\title{
New Results on Ultrafast Coherent Excitation of Molecular Vibrations in Liquids
}

\author{
W. Zinth, H.-J. Polland, A. Laubereau*, and W. Kaiser \\ Physik Department, Technische Universität, D-8000 München, \\ Fed. Rep. Germany
}

Received 24 July 1981

\begin{abstract}
Coherent Raman probe scattering experiments are performed to study dynamical processes of polyatomic liquids at $300 \mathrm{~K}$. For single homogeneous transitions the dephasing time $T_{2}$ is readily obtained from time resolved investigations. Spectral studies show an interesting time dependent shift in scattered frequency. After the excitaiton the vibrating molecules are shown to relax freely with their resonance frequency. Multiple, equally spaced transitions exhibit a beating phenomenon which provides the dephasing time and the frequency interval between neighboring vibrational states. Inhomogeneously broadened systems do not allow a ready determination of the dephasing time by the present probing technique. Previous experiments on the subject have to be reconsidered.
\end{abstract}

PACS: $33,42.80$

In a series of papers it has been demonstrated that valuable information on the dynamics of molecular vibrations in liquids may be obtained by ultrashort light pulses. Time resolved probing techniques using coherent and incoherent Raman scattering have been devised to study, respectively, phase relaxation and population life times of vibrational systems [1-4]. For a single homogeneously broadened vibrational transition the dephasing time $T_{2}$ is readily deduced from the time dependence of the coherent scattering signal. In several favorable cases the experimental $T_{2}$ values agreed well with the time constant calculated from the width of the corresponding spontaneous Raman band [1,5-7]. The situation is more complicated for closely spaced vibrational levels and for inhomogeneously broadened transitions. In several papers the time resolved coherent probe scattering has been employed to study inhomogeneously broadened transitions $[1,8-12]$. Different results were obtained depending on the experimental conditions. In a first type of experiment a rapid decay of the coherent excitation is observed resulting from the interference of states with different transition frequencies. In other experiments

^ Present address: Fachbereich Physik der Universität Bayreuth, Bayreuth, F. R. Germany with different beam geometry a slower decay of the coherent scattering signal was observed. It was assumed that the probing process sees only a small subgroup of transitions which has a dephasing time $T_{2}$. This interpretation was questioned in [1] but remained unanswered on account of insufficient information. The new experiments and the better understanding given in this paper suggest a revision of the previous interpretations of probe scattering from inhomogeneously broadened transitions.

In this paper we present a detailed discussion of transient coherent Raman probe scattering. Three different molecular systems are investigated. An isolated homogeneously broadened vibrational transition is studied first (Sect. 2). A vibrational transition consisting of several closely spaced levels is treated in Sect. 3. Inhomogeneously broadened vibrational transitions are discussed in Sect. 4.

\section{The Experimental Technique}

In our experiments we study the coherent vibrational excitation which is generated by transient stimulated Raman scattering of an ultrashort laser pulse. Only one vibration, the level with the highest Stokes gain, is 

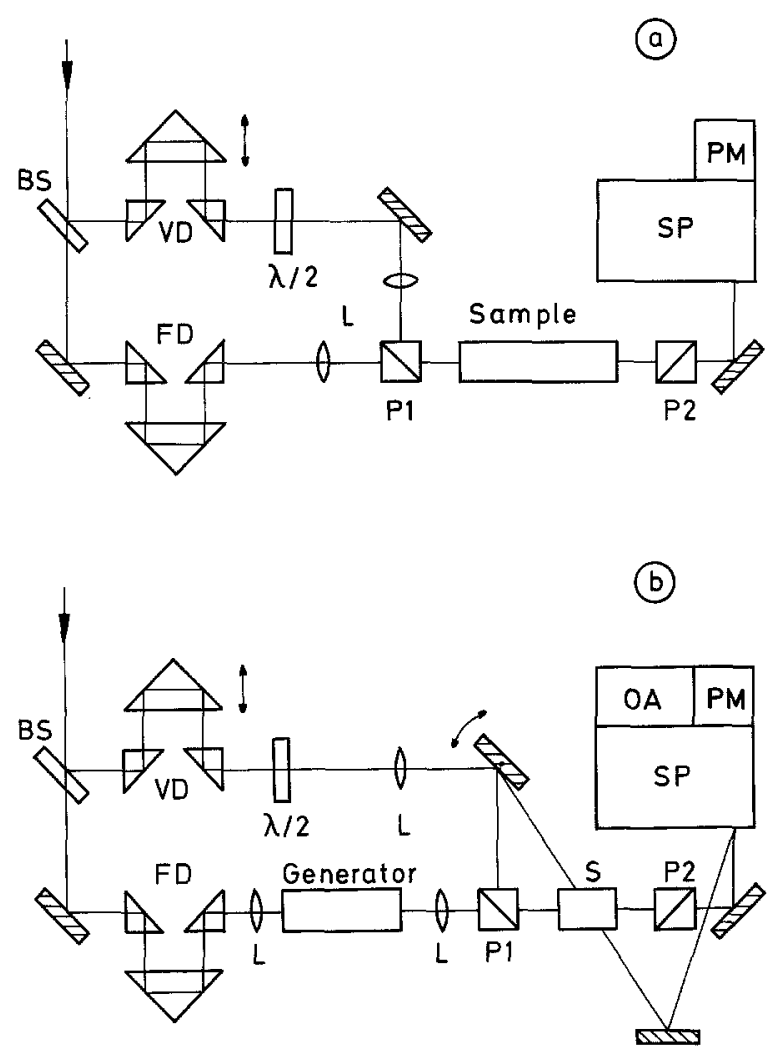

Fig. 1a and b. Schematic of two experimental systems used to study coherent probe scattering in the time and frequency domain. Beam splitter $\mathrm{BS}$, variable and fixed delay $\mathrm{VD}$ and $\mathrm{FD}$, wave plate $\lambda / 2$, lenses $\mathrm{L}$, polarizers $\mathrm{P} 1$ and $\mathrm{P} 2$ (crossed), sample $\mathrm{S}$, spectrometers $\mathrm{SP}$, photomultipliers PM, and optical multichannel analyser OA

excited. After the excitation process a weaker light pulse monitors the coherent excitation $\langle q\rangle$. The coherently scattered Stokes or anti-Stokes Raman light is detected as a function of the delay time $t_{\mathrm{D}}$. We note that the population density in the upper vibrational state $n$ is not changed significantly during the excitation process on account of the small Raman excitation cross section and the short life times in the liquids. In other words, in our investigations we deal with "small area" pulse propagation [13].

A mode-locked Nd-glass laser system supplies single ultrashort light pulses [14], which are doubled in frequency by means of a KDP crystal to $v_{\mathrm{L}} / c=18,990 \mathrm{~cm}^{-1}$. Modification of the gain bandwidth of the mode-locked laser allows to change the pulse duration between $t_{p}=3 \mathrm{ps}$ and $t_{p}=8 \mathrm{ps}$. The product, pulse duration times frequency width of the light pulses was $t_{p} \Delta v_{\mathbf{L}} \simeq 0.5$ for spectrally resolved experiments, whereas in time resolved experiments a broader spectral width of $t_{p} \Delta v_{\mathrm{L}} \simeq 1$ was used. We have employed two different experimental set-ups schematically shown in Fig. 1. In Fig. 1a the ultrashort light pulse is focussed into the sample cell generating a strong Stokes field and, simultaneously, a material excitation $\langle q\rangle$ via transient stimulated Raman scattering. The stimulated Stokes light is rejected by the polarizer P2. The probing pulse is produced by the beam splitter BS and is properly delayed in the variable delay VD. The polarization is rotated by $90^{\circ}$ using a half wave plate. The probing pulse travels collinearly with the exciting beam through the sample. The coherently scattered Stokes light passes through the polarizer P2. The spectrometer SP is used as a broadband filter at the Stokes frequency. The energy of the scattered signal is detected with the photomultiplier PM. In order to control the excitation conditions and to normalize the scattered signal we simultaneously measure the energies of the laser and the stimulated Stokes pulse. The system of Fig. 1a has some short-comings. Efficient generation of stimulated Stokes light requires a high light intensity of the laser pulse over a substantial interaction length. When exciting and probing pulses travel together through the long sample, the high intensity of the exciting pulse causes a strong phase modulation and a spectral broadening of the probing pulse. This spectral broadening caused by the nonresonant susceptibility $\chi_{\mathrm{NR}}^{(3)}$ may be drastically reduced when we separate the excitation and probing process. In the set-up of Fig. $1 \mathrm{~b}$ the stimulated Stokes pulse is generated in an extra generator cell. Stokes and laser pulses leaving this cell are imaged by the lens $L$ into the shorter sample $S$, producing the coherent material excitation to be studied. The probing pulse is introduced via the polarizer P1 and travels through the short sample $S$ collinearly with the exciting pulse. When coherent anti-Stokes scattering is observed the probing pulse crosses the exciting pulses within the sample under the welldefined phase matching angle [1]. The energy of the coherently produced light is measured by the photomultiplier PM or analyzed by a $2 \mathrm{~m}$ grating spectrograph (resolution $0.5 \mathrm{~cm}^{-1}$ ) in conjunction with an optical multichannel analyzer.

\section{Coherent Raman Scattering of a Single Homogeneously Broadened Transition}

In this section we discuss the stimulated Raman process and the coherent Raman probe scattering of a single homogeneously broadened transition. The theoretical treatment extends previous discussions on the same subject. Special attention is given to the influence of the non-resonant susceptibilities and to the development of the momentary frequencies $[1,15$, 16]. The electric field is described classically by Maxwell's equations whereas the molecules are treated quantum-mechanically [15]. The molecular transition 
is approximated by a two-level system; transitions to higher energy levels are omitted. The ensemble of noninteracting two-level systems is described by two quantities: The population density $n$ of the upper (vibrational) level and the coherent or collective vibrational amplitude $\langle q\rangle[15]$. One obtains $\langle q\rangle$ summing up the individual molecular vibrational amplitudes. For the population density $n \ll 1$ the coherent amplitude may be calculated from (1)

$$
\begin{gathered}
\frac{\partial^{2}}{d t^{2}}\langle q\rangle+\frac{2}{T_{2}} \frac{\partial}{d t}\langle q\rangle+\omega_{0}^{2}\langle q\rangle \\
=\frac{1}{2 m}\left(\frac{\partial \stackrel{\boldsymbol{\alpha}}{\hat{\imath} \mathbf{q}})(1-2 n) \mathbf{E} \mathbf{E},}{}\right.
\end{gathered}
$$

where $m$ and $\omega_{0}$ are the reduced mass and the transition frequency of the molecular vibration. The dephasing time $T_{2}$ represents the damping of the coherent amplitude. $(\partial \alpha / \partial \mathbf{q}) \mathbf{E E} / 2$ is the driving force produced by the total electric field $\mathbf{E}$ [16]. For a polarized Raman line the Raman susceptibility $(\partial \alpha / \partial q)$ is a scalar. It is interesting to see in (1) that the coherent amplitude of the molecular ensemble obeys the equation of motion of a damped oscillator coupled to the light fields by the Raman susceptibility $(\partial \alpha / \partial q)$.

The propagation of the light field $\mathbf{E}$ is governed by the wave equation:

$\nabla^{2} \mathbf{E}-\left(\frac{\mu}{c}\right)^{2} \frac{\partial^{2}}{\partial t^{2}} \mathbf{E}=\frac{4 \pi}{c} \frac{\partial^{2}}{\partial t^{2}} \mathbf{P}^{\mathrm{NL}}$.

The nonlinear interaction between light and matter is described by $\mathbf{P}^{\mathrm{NL}}$. In the stimulated Raman process we may separate $\mathbf{P}^{\mathrm{NL}}$ into a term $\mathbf{P}^{\mathrm{RES}}$ corresponding to resonant contributions and into a part $\mathbf{P}^{\mathrm{NR}}$ representing off-resonance transitions $[16,17]$.

$\mathbf{P}^{\mathrm{NL}}=\mathbf{P}^{\mathbf{R E S}}+\mathbf{P}^{\mathrm{NR}}=N\left(\frac{\partial \alpha}{\partial q}\right)\langle q\rangle \mathbf{E}+\underset{\sim}{\boldsymbol{\chi}_{\mathrm{NR}}^{(3)}} \mathbf{E E E}$,

where $N$ is the total number density of the molecules. The non-resonant interaction contains a real tensor of the third-order nonlinear susceptibility $\chi_{\mathrm{NR}}^{(3)}$.

In the following the electric fields and the coherent amplitude are assumed to be plane waves with time dependent amplitudes propagating along the $x$-axis:

$$
\begin{aligned}
& \mathbf{E}(x, t)=\frac{1}{2}\left[\sum_{j} \mathbf{E}_{j} \exp \left(\mathrm{i} k_{j} x-i \omega_{j} t\right)+\text { c.c. }\right], \\
& \langle q\rangle=\frac{1}{2}\left[Q \exp \left(i k_{q} x-i \omega_{q} t\right)+\text { c.c. }\right] .
\end{aligned}
$$

The indices $j$ refer to the incident and the produced light fields. The wave vector $k_{q}$ corresponds to the local collective vibration; it represents the phase relation between the molecules at different points in the sample. Neglecting second order derivatives of the amplitudes
$Q$ and $E_{j}$ in (1) and (2) the following equations are obtained for the transient stimulated Raman process:

$$
\begin{aligned}
& \frac{\partial Q}{\partial t}+\frac{1}{T_{2}} Q=-i \frac{1-2 n}{4 m \omega_{0}}\left(\frac{\partial \alpha}{\partial q}\right)\left(\mathbf{E}_{\mathrm{L}} \mathbf{E}_{\mathrm{S}}^{*}\right) \exp (\mathrm{i} \Delta k x), \\
& \frac{\partial \mathbf{E}_{\mathbf{S}}}{\partial x}+\frac{\mu_{\mathrm{S}}}{c} \frac{\partial \mathbf{E}_{\mathbf{S}}}{\partial t} \\
& =\frac{i \pi \omega_{\mathrm{S}}}{c \mu_{\mathrm{S}}}\left[N \frac{\partial \alpha}{\partial q} \mathbf{E}_{\mathrm{L}} Q^{*} \exp (\mathrm{i} \Delta k x)\right.
\end{aligned}
$$

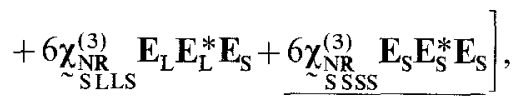

$$
\begin{aligned}
& \frac{\partial \mathbf{E}_{\mathrm{L}}}{\partial x}+\frac{\mu_{\mathrm{L}}}{c} \frac{\partial \mathbf{E}_{\mathrm{L}}}{\partial t}
\end{aligned}
$$

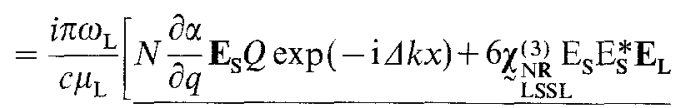

$$
\begin{aligned}
& \left.+\underset{\sim \mathrm{LR} L L}{6 \chi_{\mathrm{L}}^{(3)}} \mathbf{E}_{\mathrm{L}} \mathbf{E}_{\mathrm{L}}^{*} \mathbf{E}_{\mathrm{L}}\right],
\end{aligned}
$$

where $\mu_{\mathrm{L}}$ and $\mu_{\mathrm{S}}$ are the refractive indices at the laser frequency $\omega_{\mathbf{L}}$ and at the Stokes frequency $\omega_{\mathrm{S}}$, respectively. The nonresonant action of the field components $j, k, l$ on the component $i$ is denoted by $\underset{\sim}{\underset{N R}{\chi_{j k l}}(\mathbf{j})} \cdot{ }^{1}$ The build-up of the coherent amplitude by the laser and Stokes field is described by (6). The exponential growth of the Stokes field results from the first term on the r.h.s. of (7) and (6). $\Delta k$ is the mismatch of three wave vectors, $\Delta k=k_{\mathrm{L}}-k_{\mathrm{S}}-k_{q}$. Stimulated Raman generation requires energy conservation $\omega_{\mathrm{L}}-\omega_{\mathrm{S}}-\omega_{q}=0$; it is most effective for phase-matching, $\Delta k=0$, where the molecular excitation is prepared with the wave vector $k_{q}=k_{\mathrm{L}}-k_{\mathrm{S}}$. The excitation propagates with the velocity $v=\left(\omega_{\mathrm{L}}-\omega_{\mathrm{S}}\right) /\left(k_{\mathrm{L}}-k_{\mathrm{S}}\right)$. The second and third term on the r.h.s. of (7) influence the frequency of the Stokes field by phase modulation and self-phase modulation. The laser frequency experiences a phase modulation by the two last terms of (8). The amplitude of the laser field remains constant for a small conversion of laser energy into Stokes energy. In this case we may omit the underlined expressions in (7) and (8).

In (1)-(8) we have neglected the four-photon parametric interaction which leads to new electromagnetic

$$
\begin{aligned}
& {\underset{\sim}{\mathrm{NR}}}_{i j k l}^{(3)}={\underset{\sim}{\mathrm{NR}}}_{(3)}^{(3)}\left(\omega_{i},()_{j},-\omega_{k}, \omega_{\mathrm{L}}\right) \text { may approximately be deduced from } \\
& \text { the nonlinear refractive index } n^{2}[21] \text {; }
\end{aligned}
$$

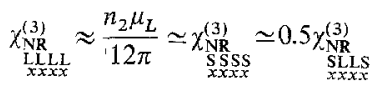




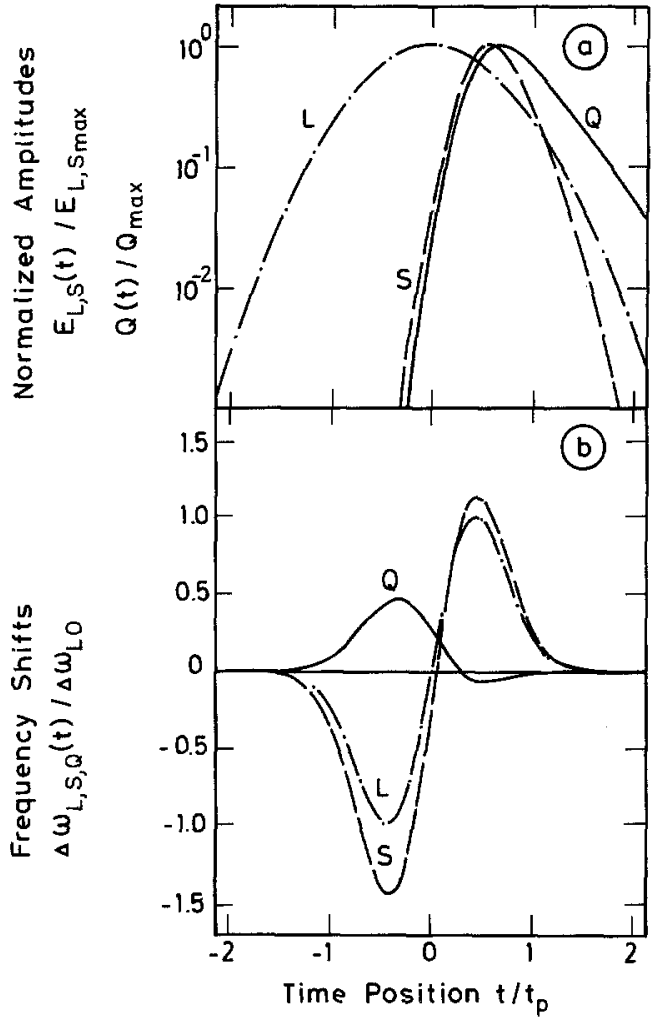

Fig. $2 \mathrm{a}$ and $\mathrm{b}$. Influence of a nonresonant susceptibility $\chi_{\mathrm{NR}}^{(3)}$ on transient stimulated Raman scattering. (a) Amplitudes of the Gaussian laser pulse L, of the Stokes field S, and of the coherent amplitude $Q$. (b) Frequency shifts induced by $\chi_{N R}^{(3)}$. At the peak of the stimulated Stokes pulse the shifts of laser and Stokes frequency are nearly equal. As a result the frequency shift of the coherent amplitude is very small. $\Delta \omega_{\mathrm{LO}}$ denotes the maximum shift of the laser frequency

waves near the Stokes and anti-Stokes frequencies. The total nonlinear susceptibility $\chi_{\mathrm{tot}}^{(3)}=\chi_{\mathrm{RES}}^{(3)}+\chi_{\mathrm{NR}}^{(3)}$ can produce light outside Raman resonances [16].

Numerical solutions of (6)-(8) have been discussed in previous publications $[24,18,19]$. We summarize here several important facts. Figure 2a shows the Gaussian shaped laser pulse (with duration $t_{p}=2.7 T_{2}$ ), the generated Stokes field $\mathbf{E}_{\mathrm{S}}$, and the coherent material excitation $Q$. The calculations were made for

$\underset{\substack{\mathrm{NR} \\ \text { LLLL } \\ x x x x}}{\chi_{(3)}^{(3)}}=1.8 \times 10^{-14} \mathrm{esu}=0.5 \times \underset{\substack{\mathrm{NR} \\ \text { SLIS }}}{(3)}$

and for a Raman gain coefficient of $g=8 \times 10^{-10} \mathrm{~cm} / \mathrm{W}$. The peaks of the stimulated Stokes field $\mathbf{E}_{\mathrm{S}}$ and of the coherent amplitude $Q$ occur delayed. While the Stokes field decays rapidly after its maximum, the coherent amplitude shows a slower exponential decay which is determined by the dephasing time $T_{2}$. We note that the time dependence of $\mathbf{E}_{\mathrm{L}}, \mathbf{E}_{\mathrm{S}}$, and $Q$ is not affected by the small value of $\left|\chi_{\mathrm{NR}}^{(3)}\right|$ employed in the calculation. But there are measurable frequency shifts which are caused by $\chi_{\mathrm{NR}}^{(3)}$ (Fig. 2b). Of importance for the stimulated Stokes process are frequency shifts occuring during the later part of the laser pulse where the generated Stokes field is large. Here, the Stokes pulse follows the frequency shift of the laser pulse and, consequently, the frequency shift of the coherent amplitude $Q$ is close to zero. At the end of the excitation process the frequency of the coherent amplitude is equal to the frequency $\omega_{0}$ of the freely oscillating molecule. The calculations of Fig. 2 are in good agreement with observations [18]: The spectra of the laser pulse and the stimulated Stokes pulse leaving the sample are broadened due to selfphase modulation. In addition, the spectrum of the Stokes pulse is shifted to higher frequencies. In liquids with relatively small values of $\left|\chi_{\mathrm{NR}}^{(3)}\right|$ Stokes shifts of several wave numbers are observed.

Information on the molecular system is obtained via coherent probing experiments. After the excitation process by a strong laser field $\mathbf{E}_{\mathrm{L}}$ a second laser pulse with $\mathbf{E}_{\mathrm{L} 2}$ enters the sample at the delay time $t_{\mathrm{D}}$. When the probe intensity is sufficiently weak, $I_{\mathbf{L} 2} \leqslant 0.1 I_{\mathrm{L}}$, the probing pulse will not influence the existing coherent amplitude. Scattered Stokes light $\mathbf{E}_{\mathrm{S} 2}$ is generated according to $(9)^{2}$

$$
\begin{aligned}
& \frac{\partial \mathbf{E}_{\mathbf{S} 2}(x, t)}{\partial x}+\frac{\mu_{\mathrm{S}}}{c} \frac{\partial \mathbf{E}_{\mathbf{S} 2}(x, t)}{\partial t} \\
& =\frac{i \pi \omega_{\mathrm{S} 2}}{c \mu_{\mathrm{S} 2}}\left\{N \frac{\partial \alpha}{\partial q} \mathbf{E}_{\mathrm{L} 2}\left(x, t-t_{\mathrm{D}}\right) Q^{*}(x, t) \exp \left(\mathrm{i} \Delta k_{1} x\right)\right.
\end{aligned}
$$

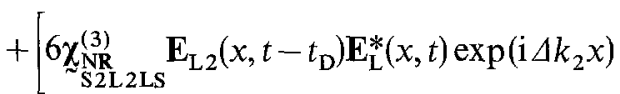

$$
\begin{aligned}
& \left.+\underset{\mathrm{S} 2 \mathrm{LL} 2 \mathrm{~S}}{6 \chi_{\mathrm{L}}^{(3)}} \mathbf{E}_{\mathrm{L}}(x, t) \mathbf{E}_{\mathbf{L} 2}^{*}\left(x, t-t_{\mathrm{D}}\right) \exp \left(\mathrm{i} \Delta k_{3} x\right)\right] \times \mathbf{E}_{\mathrm{S}}(x, t) \\
& \left.+6 \underset{\sim \mathrm{S} 2 \mathrm{LLS2}}{(3)} \mathbf{E}_{\mathrm{L}}(x, t) \mathbf{E}_{\mathbf{L}}^{*}(x, t) \mathbf{E}_{\mathrm{S} 2}(x, t)\right\} .
\end{aligned}
$$

$\Delta k_{1}=k_{\mathbf{L} 2}-k_{\mathrm{S} 2}-k_{q}$ determines the phase mismatch for the resonant part of the signal, whereas $\Delta k_{2}=k_{\mathrm{L} 2}$ $-k_{\mathrm{S} 2}-k_{\mathrm{L}}+k_{\mathrm{S}}$ and $\Delta k_{3}=k_{\mathrm{L}}-k_{\mathrm{S} 2}-k_{\mathrm{L} 2}+k_{\mathrm{S}}$ are important for the non-resonant contributions. The probing laser pulse has - in our experiments - a polarization perpendicular to the polarization of the exciting laser pulse. The Stokes light produced coherently by the isotropic Raman transition has the same polarization as the probing laser pulse and may - even in the collinear geometry - be separated from the stimulated Stokes pulse by a polarizer. Most efficient generation of scattered coherent Stokes light occurs for phase matching, $\Delta k_{1}=0$. Additional contributions to the scattered signal are produced as long as the exciting and probing laser pulses overlap in time [second term on the r.h.s. of (9)]. The non-resonant susceptibility together with $\mathbf{E}_{\mathbf{L}}$ and $\mathbf{E}_{\mathbf{L} z}$ rotate slightly the plane of

\footnotetext{
${ }^{2}$ Anti-Stokes scattering can be described by a similar equation
} 


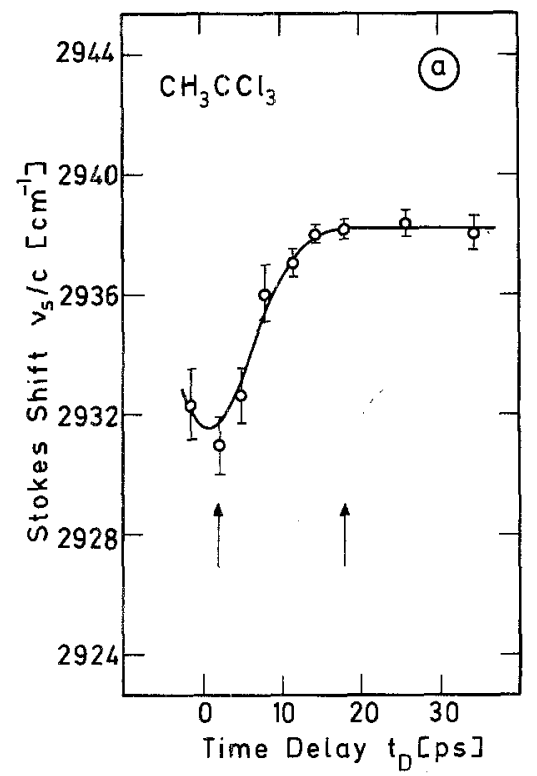

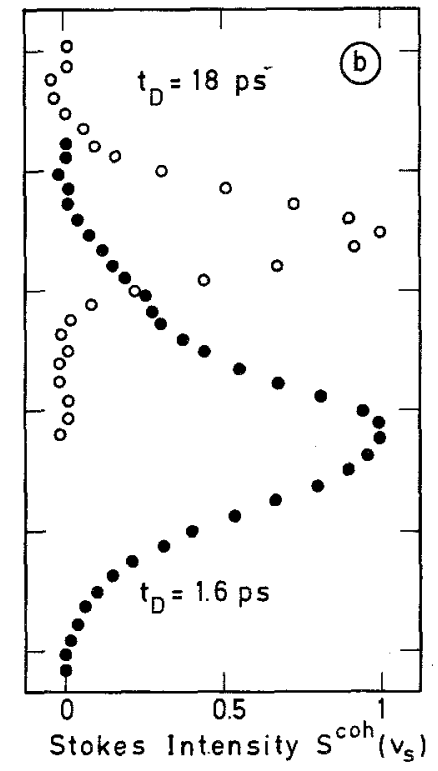

Fig. 3a and b. Experimental results of spectrally resolved coherent Raman probe scattering in liquid $\mathrm{CH}_{3} \mathrm{CCl}_{3}$ (a) Frequency of the coherently scattered Stokes light as a function of the delay time. At late times the coherent Stokes shift $v_{\mathrm{S}} / \mathrm{c}=2938.2 \mathrm{~cm}^{-1}$ is equal to the shift found in spontaneous Raman scattering $\left(2939 \mathrm{~cm}^{-1}\right)$. (b) Spectra of coherently scattered Stokes light taken with delay times $t_{\mathrm{D}}=1.6 \mathrm{ps}$ (points) and $t_{\mathrm{D}}=18 \mathrm{ps}$ (circles). Spectral broadening and frequency shift found near $t_{\mathrm{D}}=0$ are caused by the nonresonant susceptibility $\chi_{\mathrm{NR}}^{(3)}$ polarization of the stimulated Stokes light $\mathbf{E}_{\mathrm{S}}$ contributing to the observed coherently scattered light $\mathbf{E}_{\mathrm{S} 2}$. This contribution requires phase matching $\Delta k_{2}=0$ or $\Delta k_{3}=0$. The latter conditions are fulfilled for collinear geometry when the exciting and probing laser have the same frequency.

Two frequency shifts in the probing process should be mentioned here. The presence of the intense exciting laser pulse $\left(\mathbf{E}_{\mathrm{L}}\right)$ causes a frequency shift of the probing laser pulse $\left(\mathbf{E}_{\mathrm{L} 2}\right)$ and of the scattered Stokes field $\left(\mathbf{E}_{\mathrm{S} 2}\right)$ [see last term in (9)]. For later probing times $\left(t_{\mathrm{D}}>t_{p}\right)$ these phase modulations do not exist and the frequency, the magnitude and the time dependence of the coherent excitation may be studied without disturbance. Using monochromatic probing laser pulses the frequency of the coherent amplitude $Q$ is $\omega_{q}=\omega_{\mathrm{L} 2}-\omega_{\mathrm{S} 2}$. The time development of $Q$ is obtained when the energy of the scattered Stokes pulse, $S^{\mathrm{coh}}\left(t_{\mathrm{D}}\right)$, is measured as a function of delay time $[1,19]$ :

$$
\begin{aligned}
S^{\mathrm{coh}}\left(t_{\mathrm{D}}\right) & \propto \int d t\left|E_{\mathrm{S} 2}\right|^{2} \\
= & \int_{-\infty}^{+\infty} d \tilde{t} \mid \int_{0}^{L} d \tilde{x} \frac{\mathrm{i} \pi \omega_{\mathrm{S} 2}}{c \mu_{\mathrm{S} 2}} N \frac{\partial \alpha}{\partial q} \\
& \cdot \mathbf{E}_{\mathrm{L} 2}\left(\tilde{x}, \tilde{t}-t_{\mathrm{D}}\right) Q^{*}(\tilde{x}, \tilde{t}) \times\left.\exp \left(\mathrm{i} \Delta k_{1} \tilde{x}\right)\right|^{2} .
\end{aligned}
$$

Equation $(9 a)$ is written for the moving frame with $\tilde{x}=x$ and $\tilde{t}=t-\frac{x}{c} \mu_{\mathrm{s} 2}$. The non-resonant susceptibility is neglected in $(9 \mathrm{~b})$. The coherent signal reflects the squared convolution between the probing laser pulse $\mathbf{E}_{\mathrm{L} 2}$ and the coherent amplitude $Q$. For sufficiently short laser pulses, $t_{p} \leqq T_{2}$, we may deduce the dephasing time $T_{2}$ from the decay of the scattered signal
$S^{\mathrm{coh}}\left(t_{\mathrm{D}}\right)$. When the non-resonant susceptibilities are large, additional stimulated Stokes signals may be generated when pump and probe pulses are simultaneous in the sample. The dephasing time may be obtained when scattered Stokes signals are considered at late delay times [17].

As an example we present investigations of the coherent Stokes scattering in liquid 1,1,1-trichloroethane $\left(\mathrm{CH}_{3} \mathrm{CCl}_{3}\right)$ at room temperature. The symmetrical $\mathrm{CH}_{3}$-stretching mode at $v / c=2,939 \mathrm{~cm}^{-1}$ has a Lorentzian shape in the spontaneous Raman spectrum with spectral width of $4.3 \mathrm{~cm}^{-1}$. In the probing experiments we used the set-up shown in Fig. 1b. The lengths of the generator and sample cells were $5 \mathrm{~cm}$ and $1 \mathrm{~cm}$, respectively. Both cells contained the same liquid. The conversion of laser to Stokes energy was approximately $5 \%$. We used bandwidth limited laser pulses with a duration of $t_{p}=6.5 \mathrm{ps}$. The time resolved probing experiments show an exponential decay of the Stokes signal with a time constant $\tau=T_{2} / 2=1.3 \pm 0.2 \mathrm{ps}$ in agreement with the spontaneous Raman width. In more recent experiments we studied the spectra of the coherently scattered Stokes and anti-Stokes light. In Fig. 3a we plotted the peak frequencies of the coherent Stokes spectra as a function of the delay time $t_{\mathrm{D}}$; in Fig. $3 b$ two corresponding spectra at early and late probing times are depicted. At $t_{\mathrm{D}}=0$ both the exciting and the probing pulse travel together through the sample. We find for this situation a large shift of the scattered Stokes light to higher frequencies (i.e., a smaller Stokes shift). In addition, Fig. $3 b$ shows a broad spectrum for small delay times. The observed Stokes signal consists of two contributions: Coherently scattered Stokes light $\left(\mathbf{E}_{\mathrm{S} 2}\right)$ [frequency 


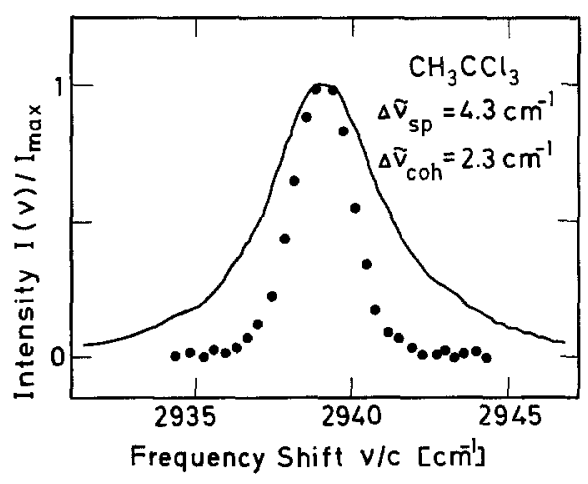

Fig. 4. Transient coherent spectrum (points) and spontaneous Raman spectrum (solid curve) of the symmetric $\mathrm{CH}_{3}$-stretching mode of liquid $\mathrm{CH}_{3} \mathrm{CCl}_{3}$. Both spectra show the same Raman shift but different spectral widths of $4.3 \mathrm{~cm}^{-1}$ (spontaneous scattering) and $2.3 \mathrm{~cm}^{-1}$ (transient coherent scattering)

shifted according to the last term on the r.h.s. of (9)] and stimulated Stokes light $\left(\mathbf{E}_{\mathrm{s}}\right)$ which experienced a rotation of polarization [second term in (9)] and which has a phase modulation according to (7).

Of special interest are our results at later delay times, $t_{\mathrm{D}} \geqq 18 \mathrm{ps}$, when the strong exciting pulse has left the sample. As seen in Fig. 3a, the scattered Stokes frequency reaches a constant value. The experimentally found vibrational frequency $\omega_{q}=\omega_{\mathrm{L} 2}-\omega_{\mathrm{S} 2}$ of $2.938 .2 \pm 0.5 \mathrm{~cm}^{-1}$ is very close to the value of $2,939.0 \pm 0.5 \mathrm{~cm}^{-1}$ found in spontaneous Raman experiments; i.e. the coherently excited molecules vibrate with their resonance frequency. We point to the narrow spectrum at $t_{\mathrm{D}}=18 \mathrm{ps}$ seen in Fig. $3 \mathrm{~b}$. The spectrum has a smaller halfwidth than the spontaneous Raman band. This surprising observation is shown more clearly in Fig. 4 where a spontaneous Raman spectrum (solid line) is compared with a coherent antiStokes probing spectrum taken with a delay time of $18 \mathrm{ps}$ (points). The spontaneous band width of $4.3 \mathrm{~cm}^{-1}$ is nearly a factor of two wider than the coherent spectrum of $2.3 \mathrm{~cm}^{-1}$. We note that the latter spectrum has the same width as the probing laser pulse. For an explanation of this observation we recall the properties of the probing process. The coherently excited amplitude $Q$ decays exponentially with time. The coherent scattering signal $\left(S^{\text {coh }}\right)$ is a convolution of the Gaussian probe pulse with the exponential decay of $Q$, see (9a). As a result, $S^{\text {coh }}$ has the same spectral width as the probe pulse and may be smaller than the spontaneous Raman band. For long probe pulses and long interaction with the exponentially decaying coherent amplitude one obtains a small width of the coherent scattering signal. This technique may help to determine the vibrational frequency with improved accuracy and may be useful to resolve closely lying vibrational transitions.

\section{Multiple Equidistant Homogeneously Broadened Transitions}

Vibrational modes consisting of several equally spaced homogeneous transitions are discussed in this section. We study molecular vibrations where the varying isotop compositions give rise to slightly different resonance frequencies $\omega_{j}$ with equal frequency differences $\Delta \omega=\omega_{j}-\omega_{i}$. The spontaneous line width of the individual transitions $\delta \omega_{j}$ and the frequency difference $\Delta \omega$ are both small compared to $\omega_{j}$. The theoretical treatment of such a system follows closely the discussion of a single homogeneous transition (Sect. 2). Each component $j$, occuring with number density $N_{j}$, has a coherent amplitude $q_{j}$. We define a total coherent amplitude $\left\langle q_{\text {tot }}\right\rangle=\sum_{j} N_{j}\left\langle q_{j}\right\rangle$. To simplify the discussion we assume that the different molecular species have the same dephasing time $T_{2}$. With the ansatz of plane waves and slowly varying envelopes for the coherent amplitude and the electric fields - (4) and (5) we obtain the following set of equations for the transient stimulated Raman process $[1,8,16]$ :

$$
\begin{aligned}
\frac{\partial Q_{j}}{\partial t}+\frac{1}{T_{2}} Q_{j}= & -i \frac{1-2 n}{4 m\left(\omega_{\mathrm{L}}-\omega_{\mathrm{S}}\right)}\left(\frac{\partial \alpha}{\partial q}\right)\left(\mathbf{E}_{\mathrm{L}} \mathbf{E}_{\mathrm{S}}^{*}\right) \\
& \cdot \exp \left(i \Delta k_{j} x-\mathrm{i} \Delta \omega_{j} \mathrm{t}\right)
\end{aligned}
$$

$$
\begin{aligned}
& \frac{\partial \mathbf{E}_{\mathbf{S}}}{\partial x}+\frac{\mu_{\mathrm{S}}}{c} \frac{\partial \mathbf{E}_{\mathbf{S}}}{\partial t} \\
& =\frac{\mathrm{i} \pi \omega_{\mathrm{S}}}{c \mu_{\mathrm{S}}}\left[\sum_{j} N_{j} \frac{\partial \alpha}{\partial q} \mathbf{E}_{\mathrm{L}} Q_{j}^{*} \exp \left(\mathrm{i} \Delta k_{j} x-\mathrm{i} \Delta \omega_{j} t\right)\right.
\end{aligned}
$$

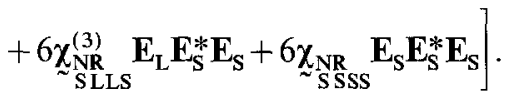

An additional equation describing the laser field is obtained when we replace $N \times Q \times \exp (-\mathrm{i} \Delta k x)$ in (8) by the sum $\sum_{j} N_{j} Q_{j} \times \exp \left[\mathrm{i}\left(\Delta \omega_{j} t-\Delta k_{j} x\right)\right]$. As in the case of a single vibrational component, most efficient Stokes amplification is found when phase matching takes place, i.e., when $\Delta k_{j}=k_{\mathrm{L}}-k_{\mathrm{S}}-k_{q}=0$. In the following discussion the non-resonant susceptibility is neglected. For very short laser pulses one has to consider the transient Raman excitation of the various vibrational species. A detailed analysis of (10) and (11) shows that in the transient case the vibrational components are excited to nearly the same coherent amplitude and all molecules vibrate at the frequency of the driving force $\omega_{0}=\omega_{\mathrm{L}}-\omega_{\mathrm{S}}$. There is one stimulated Stokes wave with frequency $\omega_{\mathrm{S}}=\omega_{\mathrm{L}}-\omega_{0}=\omega_{\mathrm{L}}$ $-\sum \omega_{j}\left(N_{j} / N\right)$, where $N$ is the total number density of the molecules. The coherent excitation has the 
wave vector $k_{q}=k_{\mathrm{L}}-k_{\mathrm{S}}$ and propagates with the velocity $\left.\left(\omega_{\mathrm{L}}-\omega_{\mathrm{S}}\right) / \mathrm{k}_{\mathrm{L}}-\mathrm{k}_{\mathrm{S}}\right)$. When the excitation process terminates the different vibrational components return to their resonance frequencies $\omega_{j}$. The frequency changes $\Delta \omega_{j}=\omega_{j}-\omega_{0}$ lead to a simultaneous change of wave vector $\delta k_{j}=\Delta \omega_{j} / v^{\prime}$, when the driving force leaves the sample with the velocity $v^{\prime}=c / \mu_{\mathrm{S}}$. We emphasize the fact that the different molecular components vibrate with a common wave vector during the excitation but have individual wave vectors $k_{j}$ during the free relaxation. Since the molecules were coherently excited, they start their relaxation with fixed phases. Interference is expected between the different components influencing the decay of the total coherent amplitude. In our experiment with several equidistant transition frequencies one finds destructive and constructive interference with a recurrence time of the maxima $t_{M}=2 \pi / \Delta \omega$ where $\Delta \omega=\omega_{j}-\omega_{i}$ has been defined above.

In the experiment one detects the energy of the scattered Stokes light $S^{\mathrm{coh}}\left(t_{\mathrm{D}}\right)$ generated by the delayed probing laser pulse $\mathbf{E}_{\mathrm{L} 2}$ of perpendicular polarization. We neglect the influences of $\chi_{\mathrm{NR}}^{(3)}$ and calculate $S^{\mathrm{coh}}\left(t_{\mathrm{D}}\right)$ in the moving frame of the Stokes light $\tilde{x}=x$ $\tilde{t}=t-x \mu_{\mathrm{S} 2} / c$ :

$$
\begin{aligned}
& S^{\mathrm{coh}}\left(t_{\mathrm{D}}\right) \\
& \propto \int_{-\infty}^{+\infty} d \tilde{t} \mid \int_{0}^{L} d \tilde{x} \sum_{j} \mathbf{E}_{\mathrm{L} 2}^{*}\left(\tilde{t}-t_{\mathrm{D}}\right) \times N_{j} Q_{j}(\tilde{x}, \tilde{t}) \\
& \left.\quad \cdot \exp \left(\mathrm{i} \Delta k_{j} \tilde{x}-\mathrm{i} \Delta \omega_{j} \tilde{t}\right)\right|^{2} .
\end{aligned}
$$

For an exponential growth of the Stokes light over the cell length $L, E_{\mathrm{S}}(\tilde{x})=E_{\mathrm{S}}(0) \exp \left(\frac{\tilde{x}}{2 A l}\right)$, we obtain from (12):

$$
\begin{aligned}
& S^{\mathrm{coh}}\left(t_{\mathrm{D}}\right) \\
& \propto \int_{-\infty}^{+\infty} d \tilde{t} E_{\mathrm{L} 2}^{2}\left(\tilde{t}-t_{\mathrm{D}}\right) \mid \sum_{j} N_{j} Q_{j}(L, \tilde{t}) \exp \left(-\mathrm{i} \Delta \omega_{j} \tilde{t}\right) \\
& \quad \cdot\left[1+\left(2 \Delta l \Delta k_{j}\right)^{2}\right]^{-1 / 2} \\
& \left.\quad \cdot \exp \left[\mathrm{i} \Delta k_{j} L+i \arctan \left(2 \Delta l \Delta k_{j}\right)\right]\right|^{2},
\end{aligned}
$$

where $\Delta k_{j}$ is the phase mismatch for each vibrational component $\Delta k_{j}=k_{\mathrm{L} 2}-k_{\mathrm{s} 2 j}-k_{j}=\Delta k_{\mathrm{s} 20}+\delta k_{\mathrm{s} 2 j}$, and $\Delta k_{\mathrm{S} 20}$ denotes the phase mismatch of the components at the center frequency. According to (13) the coherent signal is small for $\Delta k_{j} \Delta l>1$; i.e., for a large phase mismatch $\Delta k_{j}$ and a long interaction length $\Delta l$. In practical cases phasematching is established for the vibrational component in the center of the frequency distribution, i.e., $\Delta k_{\mathrm{S} 20}=0$. Under these conditions all vibrational components contribute to the coherent signal during the excitation process (where $\delta k_{\mathrm{s} 2 j}=0$ ). After the excitation the components vibrate with their individual frequencies $\omega_{j}=\omega_{0}-\Delta \omega_{j}$ and a phase mismatch $\delta k_{j}=\Delta \omega_{j} / c\left(\mu_{\mathrm{S}} \cos \alpha-\mu_{\mathrm{S} 2 j}\right)$ occurs. $\alpha$ is the angle between the exciting and the scattered Stokes beams, $\mu_{\mathrm{S} 2 j}$ is the refractive index at the scattered Stokes frequency $\omega_{S 2 j}=\omega_{\mathbf{L} 2}-\omega_{j}$. When we use the same laser frequency for the excitation and for the probing process, $\omega_{\mathrm{L}}=\omega_{\mathrm{L} 2}$, and a collinear geometry, the mismatch $\delta k_{j}$ is very small: For instance, $\delta k_{f} \simeq 10^{-4} \mathrm{~cm}^{-1}$ is calculated for $\mathrm{CCl}_{4}$ at $\omega_{L} / 2 \pi c=19,000 \mathrm{~cm}^{-1}$ and $\Delta \omega_{j} / 2 \pi c$ $=3 \mathrm{~cm}^{-1}$. Somewhat larger values of $\delta k_{j}$ are possible when different laser frequencies $\omega_{\mathbf{L}} \neq \omega_{\mathbf{L} 2}$ and a strongly dispersive medium are used. In this case, however, the interaction length $\Delta l$ is limited by velocity dispersion.

We conclude from this discussion that $\Delta k_{j} \Delta l<1$ holds for all vibrational components; the individual observation of one vibrational component by selective phase matching does not appear to be feasible.

To see the salient properties of the transient Raman excitation of multiple transitions we calculated solutions of (10)-(12) for the case of small Stokes conversion. We considered a system of four vibrational transitions with the relative number densities $N_{1} / N=32.5 \%, \quad N_{2} / N=42.2 \%, \quad N_{3} / N=20.5 \%, \quad$ and $N_{4} / N=4.4 \%$ with equal dephasing times $T_{2}$ and with a separation $\Delta \omega T_{2}=3.77$. These data are relevant for the $v_{1}$-stretching mode at $v / c \simeq 459 \mathrm{~cm}^{-1}$ of liquid $\mathrm{CCl}_{4}$ and will be compared with experimental results below. Calculations were made for two pulse durations $t_{p}=1.33 T_{2}$ and $t_{p}=0.58 T_{2}$ and for Gaussian shaped laser pulses. The nonresonant susceptibility is neglected. The starting condition of the Stokes field is spontaneous Raman light with randomly distributed phases. Our calculation of the electric field is performed classically. A recent quantum mechanical treatment [20] gives essentially the same results in the high amplification limit.

Our theoretical results are summarized as follows: During the excitation process the stimulated Stokes pulse is generated delayed similar to the case of a single homogeneous transition (Fig. 2a). The instantaneous frequencies and the coherent amplitudes of the four transitions show a pronounced time dependence. In Fig. 5, calculated curves are presented for two pulse durations. During the rising part of the exciting laser pulse (i.e. for times $t / T_{2}<0$ ) the coherent vibrational components grow rapidly over several orders of ten. The coherent amplitude continues to increase by approximately a factor of one hundred after the peak of the exciting pulse has passed the considered volume (Fig. $5 \mathrm{c}$ and d). We see from Fig. $5 \mathrm{a}$ and $\mathrm{b}$ that at early times the various vibrational components are driven with nearly the same frequency. After the peak of the 


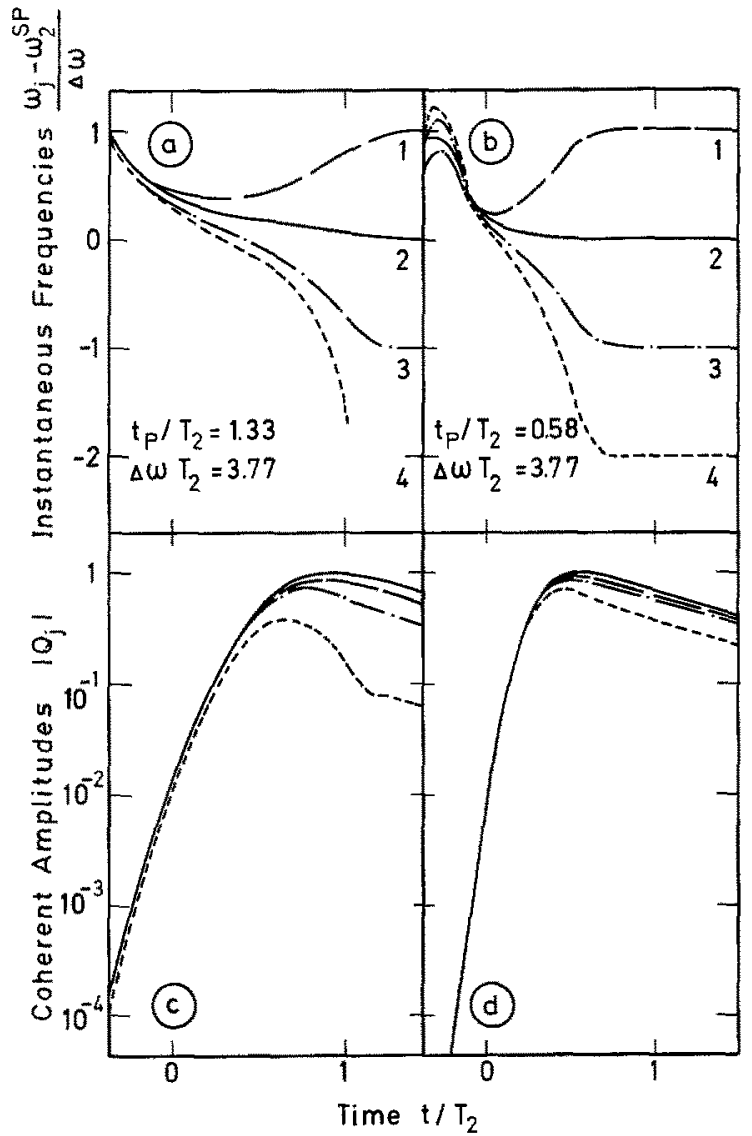

Fig. 5a-d. Time development of instantaneous frequencies (upper part) and of coherent amplitudes (lower part) calculated for four homogeneously broadened equidistant transitions (1 to 4) after excitation by a Gaussian shaped laser pulse with pulse durations $t_{p}=1.33 T_{2}$ (1.h.s.) and $t_{p}=0.58 T_{2}$ (r.h.s.). Longer pulses show a later return to the resonant frequencies and a larger difference between the four coherent amplitudes. $\omega_{2}^{\mathrm{sP}}$ denotes the spontaneous frequency of the most abundant component 2

excitation pulse the vibrational frequencies begin to return to their resonance values. As a result, the various components get out of phase to the driving force and the growth of the coherent amplitude terminates. We point to the effect of longer pulse duration: The coherent amplitude of component 4 is substantially reduced (Fig. 5c) when a phase shift of approximately $\pi$ occurs between the driving force and the coherent amplitude. It should be recalled that in the stationary stimulated Raman process the most abundant component $2(42.2 \%)$ is much more excited than the following component $1(32.5 \%)$; for a Stokes amplification of $10^{10}$ one readily estimates an enhancement factor 10 . Completely different is the strongly transient case (Fig. 5 d) where the coherent amplitudes of all the four components grow to very similar amplitudes.

The total coherent amplitude $\left|Q_{\text {tot }}\right|^{2}-$ i.e., the superposition of the amplitudes of the four components - is

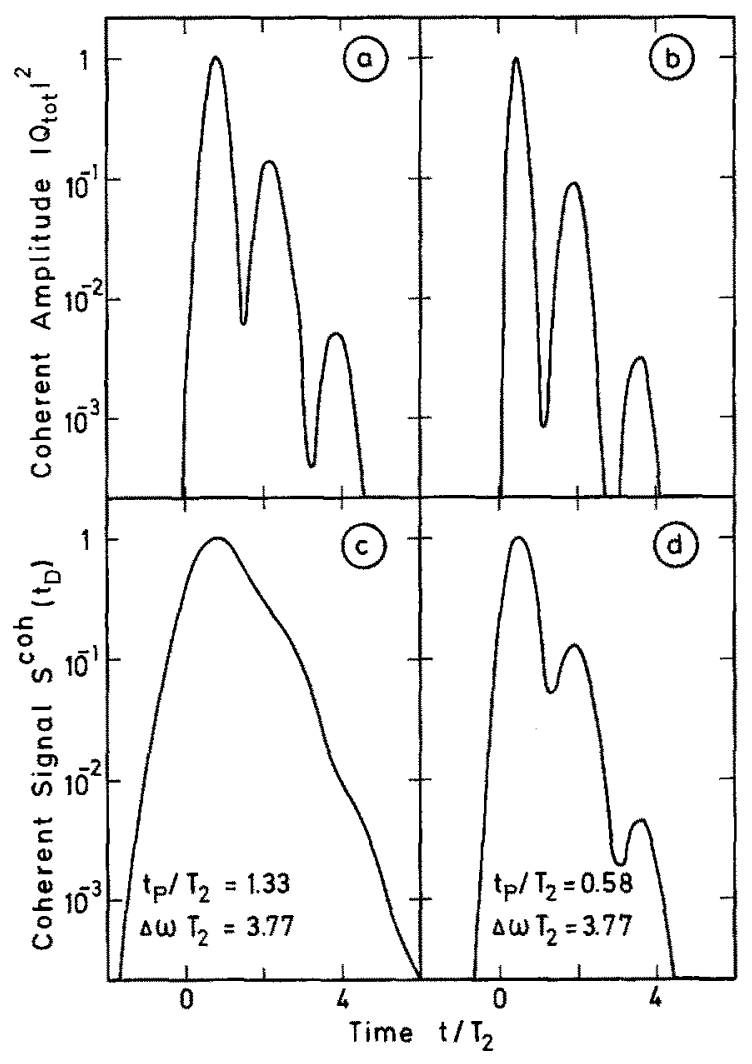

Fig. 6a-d. Calculated time development of the total coherent amplitude $\left|Q_{\text {tot }}\right|^{2}=\left|\sum_{j} N_{j} Q_{j} \exp \left(\mathrm{i} \Delta \omega_{j} t\right)\right|^{2}$ (upper part) and the coherent probe signal $S^{\text {coh }}\left(t_{\mathrm{D}}\right)$ (lower part) for different durations of the laser pulses $t_{p}=1.33 T_{2}$ (l.h.s.) and $t_{p}=0.58 T_{2}$ (r.h.s.). Note the smooth decay of the signal for the longer pulses and the pronounced beating structure for the shorter pulses

depicted in Fig. $6 a$ and $b$ for two pulse durations. As predicted in the previous discussion, the different vibrational components (with frequency difference $\Delta \omega$ ) get out of phase and generate a distinct beating pattern. In addition, the total amplitude decays exponentially due to the dephasing of the different coherent amplitudes. We note that the modulation is less pronounced for longer pulses since the more distant components are excited less efficiently (Fig. $5 \mathrm{c}$ ). In the lower part of Fig. 6 we plotted the coherent signal obtained by probing the total coherent amplitude with a laser pulse having the same duration as the exciting pulse. The two curves of Fig. $6 \mathrm{c}$ and $\mathrm{d}$ exhibit a completely different pattern: The longer probing pulses (Fig. 6c) have smeared out the beating structure still found in the plot of the coherent amplitude (Fig. 6a). The decay of the coherent signal shows a small modulation. From the envelope one may deduce the dephasing time $T_{2}$. The envelope of the decay of the 


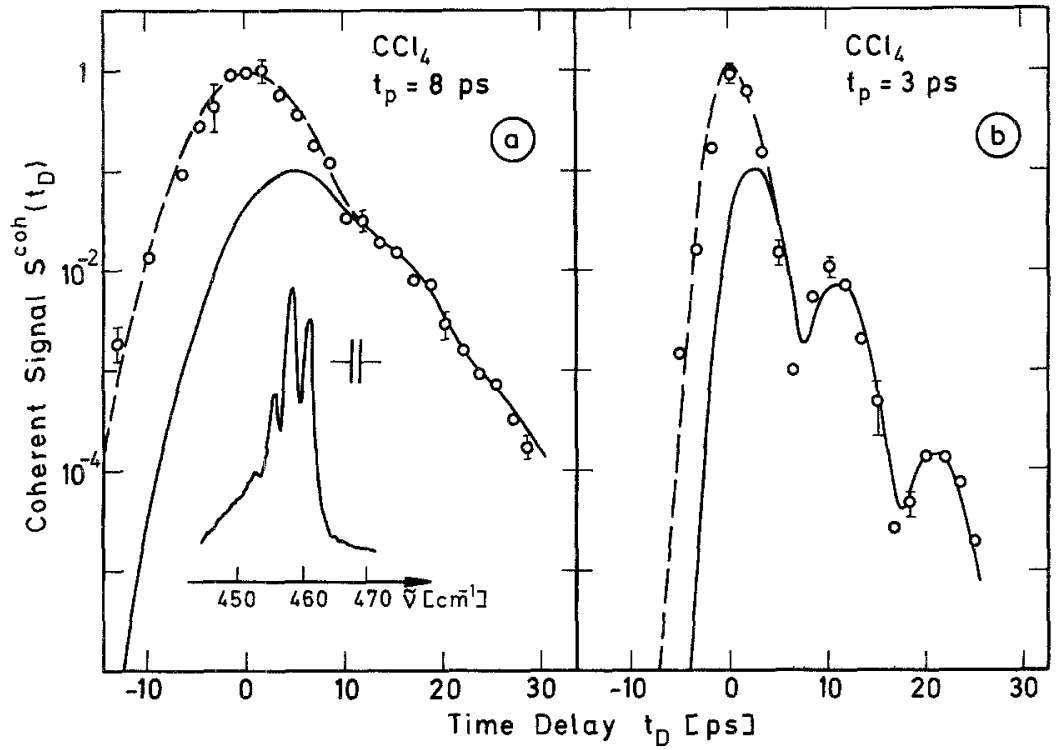

Fig. $7 \mathrm{a}$ and $\mathrm{b}$. Experimental results of time resolved coherent Raman probe scattering on the symmetric mode of $\mathrm{CCl}_{4}$ at $459 \mathrm{~cm}^{-1}$; insert: Raman spectrum. Two different durations of the exciting and probing laser pulses are used: $t_{p}=8 \mathrm{ps}$ (a) and $t_{p}=3 \mathrm{ps}$ (b). Both curves allow the determination of the dephasing time $T_{2}$. The additional signal near $t_{\mathrm{D}}=0$ (broken curve) is attributed to nonresonant light scattering signal intensity decreases with a time $\tau=T_{2} / 2$. When the exciting and probing pulses are shorter by a factor of 2.3 only, the beating is clearly resolved (Fig. 6d). From the beating period one may calculate the frequency distance of the vibrational components. From the decay of the envelope of the curve the dephasing time $T_{2}$ may be deduced.

Liquid $\mathrm{CCl}_{4}$ was found to be an ideal medium for the experimental investigation of coherent Raman probe scattering from multiple transitions. The spontaneous Raman spectrum of $\mathrm{CCl}_{4}$ at $459 \mathrm{~cm}^{-1}$ (insert of Fig. 7) consists of four peaks with a distance of $3 \mathrm{~cm}^{-1}$. The different transition frequencies are caused by the chlorine isotopes ${ }^{37} \mathrm{Cl}$ and ${ }^{35} \mathrm{Cl}$ in $\mathrm{CCl}_{4}[8]$. The experiments were performed with the generator set-up of Fig. 1a. We carefully controlled the duration of the laser pulses measuring the autocorrelation function before and after each measurement. The laser intensity was adjusted making the conversion of laser energy into Stokes energy small, approximately $5 \%$. Two representative results are shown in Fig. 7. The coherent scattering signal is plotted as a function of the delay time $t_{\mathrm{D}}$. Time zero was determined by the peak of the autocorrelation curve with an accuracy of better than 1 ps. The experimental points are averaged over more than 15 individual measurements. The liquid cell has a length of $10 \mathrm{~cm}$. Figure $7 \mathrm{a}$ shows data taken with pulses of a mean duration of $8 \mathrm{ps}$. The signal curve rises to its maximum at $t_{\mathrm{D}}=0$ and decays subsequently approximately exponentially with a time constant of close to $3 \mathrm{ps}$. The small modulation superimposed on the exponential decay is only seen in very careful measurements. Using the same geometry but shorter light pulses with a mean duration of $t_{p}=3 \mathrm{ps}$, a completely different pattern of the signal curve is found. A pronounced modulation with distinct minima at
$t_{\mathrm{D}}=6 \mathrm{ps}$ and $t_{\mathrm{D}}=17 \mathrm{ps}$ is depicted in Fig. $7 \mathrm{~b}$. The first peak of the signal curve is located at time zero. The solid curves in Fig. $7 \mathrm{a}$ and $\mathrm{b}$ are calculated with parameters relevant for $\mathrm{CCl}_{4}$; the procedure has been discussed above and the corresponding curves (in normalized units) were shown in Fig. 6c and d. We see from Fig. 7 that both calculated curves fit the experimental points very well for $t_{\mathrm{D}}>10$; i.e., for the time range of free relaxation of the molecular ensemble. The experimental data provide the dephasing time $T_{2}=6.0 \pm 0.4 \mathrm{ps}$ and the beating interval gives the frequency difference of two neighboring components $\Delta \omega / 2 \pi c=3.1 \pm 0.2 \mathrm{~cm}^{-1}$ in agreement with spontaneous Raman measurements.

Figure 7 shows for delay times $t_{\mathrm{D}} \lesssim 0$ a considerably larger scattering signal than predicted from the theory of stimulated Raman scattering. Obviously, the additional scattering component is of different physical origin. A clue for the interpretation comes from the following two experimental observations. First, the extra signal is directly related to the laser pulse which peaks at $t_{\mathrm{D}}=0$. Adding to the solid curve a term proportional to the laser pulse, one obtains the broken lines which fit the experimental data perfectly. Second, the spectrum of the scattered signal was measured at $t_{\mathrm{D}}=0$ and found quite broad, approximately $30 \mathrm{~cm}^{-1}$. This spectrum differs strongly from the narrow spectra obtained from the later scattered pulses at $t_{\mathrm{D}}>5 \mathrm{ps}$. We strongly feel that the additional scattering signal is generated by parametric four-photon interaction. This process is fast, follows the laser pulse at saturation, and its spectrum is not sharply peaked at the Raman frequency.

A series of additional experiments was carried out to obtain a better understanding of the coherent Raman probing of $\mathrm{CCl}_{4}$. We varied the interaction length of, 
the coherent excitation with the probing pulse between $1 \mathrm{~mm}$ and $10 \mathrm{~mm}$ using various cell lengths and the setup of Fig. 1b. We also changed the ratio of probe to pump intensity from $10^{-1}$ to $10^{-3}$.

In all these experiments we obtained results very similar to those shown in Fig. 7. Slight variations were found when the intensity of the exciting laser pulse, i.e. the degree of Stokes conversion, was increased. At high Stokes conversions, where saturation takes place, the beating structure is less pronounced but still resolvable. Several collinear measurements were made with probing on the anti-Stokes side of the spectrum. In spite of considerable phase mismatch a high signal at $t_{\mathrm{D}}=0$ was observed corresponding to a four-photon parametric process. The coherent signal at later delay times was reduced according to the phase mismatch. The well resolved beating was always found when pulses with short duration were used.

The experimental results demonstrate that phase matching is non-selective. All the excited components contribute to the signal giving an interference phenomena. The results of the various experiments agree well with the calculated solutions of (10)-(12).

A comment should be made on earlier publications on coherent scattering of the $459 \mathrm{~cm}^{-1}$ vibration of liquid $\mathrm{CCl}_{4}[7,8]$. A modulated scattering curve was observed in a collinear geometry with short interaction length. The beating phenomena and the signal decay were interpreted correctly. In a different experimental set-up with a longer cell length, a smooth unmodulated decay of the scattering signal was reported. It was thought that in the latter experiment a single component of the multiple transition was probed with the help of selective phase matching. This interpretation may not be maintained in the light of our present experimental and theoretical understanding. In our opinion, accidentally, longer laser pulses and a higher Stokes conversion are responsible for the observed smooth decay of the scattered probe signal.

\section{Continuously Distributed Vibrational Frequencies}

Inhomogeneously broadened transitions with continuously distributed resonances are frequently found in liquids. The interaction of a molecule with its specific surrounding is responsible for slight changes of the resonance frequencies. The spontaneous Raman spectra have no longer a Lorentzian shape and, in some cases, the bands become quite broad exceeding several tens of wave numbers. The dephasing time $T_{2}$ can no longer be determined from the half-width of the spontaneous band.

We introduce a distribution function $N(\Delta \omega)$ for the inhomogeneously broadened systems. $\Delta \omega$ is the difference between the center frequency $\omega_{0}$ and the specific resonance frequency. $N(\Delta \omega)$ is normalized giving the total number density of molecules $N=\int_{-\infty}^{+\infty} N(\Delta \omega) d \Delta \omega$. Stimulated Raman excitation and coherent Raman probe scattering may be described by (10)-(12) when $N_{j}$ is replaced by $N(\Delta \omega), Q_{i}$ by $Q(\Delta \omega)$, and $\Delta \omega_{j}$ by $\Delta \omega$. The summation over the index $j$ has to be replaced by the integration over $\Delta \omega$. The dephasing time $T_{2}$ is assumed to be constant over the entire spectral bandwidth and the width $D \Omega$ for the inhomogeneous distribution is large with $D \Omega \gg 1 / t_{p}$. A detailed theoretical study was made of this system; the salient properties are: The stimulated Stokes pulse grows rapidly with the input pulse and the peak of the Stokes light coincides with the peak of the exciting laser pulse. The generated Stokes pulse is much shorter than the laser pulse. The Stokes frequency $\omega_{\mathrm{S}}$ corresponds to the maximum of the inhomogeneous distribution (when $\chi_{\mathrm{NR}}^{(3)}$ is neglected). The coherent vibrations are driven during the excitation process at the center frequency $\omega_{0}=\omega_{\mathrm{L}}-\omega_{\mathrm{S}}$. Neighboring frequencies are excited within a bandwidth $d \Omega$ determined by the bandwidth of the driving force (proportional to $\mathbf{E}_{\mathrm{L}} \mathbf{E}_{\mathrm{S}}$ ). In general, $d \Omega$ is determined by the short duration $t_{p s}$ of the Stokes pulse; i.e., $d \Omega \simeq 1 / t_{p s}$. When the exciting force vanishes the vibrating molecules return to their resonance frequencies and the coherent amplitudes at each frequency $\omega_{0}+\Delta \omega$ decay with the dephasing time $T_{2}$. Calculating the total coherent amplitude $Q_{\text {tot }}=\int d \Delta \omega Q(\Delta \omega) N(\Delta \omega)$ we find a destructive interference between the coherent amplitudes at different frequency positions. The total coherent amplitude drops with a time of approximately $1 / d \Omega$. We have seen in the previous chapter that the coherent probe signal corresponds to the convolution of the total coherent amplitude with the probing laser pulse. Since the coherent amplitude exists for a very short time (approximately for the duration of the short stimulated Stokes pulse) the time dependence of the coherent signal will reproduce the shape of the probing laser pulse. This fact has been demonstrated in an earlier publication [22]. It is important to point out that selective phase matching of a molecular subgroup (suggested previously) cannot be realized in coherent probing experiments.

We have made numerous experimental investigations using liquid methanol and acetone. Methanol has two strong Raman bands at $2835 \mathrm{~cm}^{-1}$ and $2940 \mathrm{~cm}^{-1}$ (CH-stretching modes) which are inhomogeneously broadened with spectral widths exceeding $20 \mathrm{~cm}^{-1}$. The stimulated Raman process occurs at either one of the two transitions when working at small Stokes conversion of approximately $5 \%$. Using a spectrograph and an optical multichannel analyser we monitored the generated Stokes frequency at each laser pulse. In a 


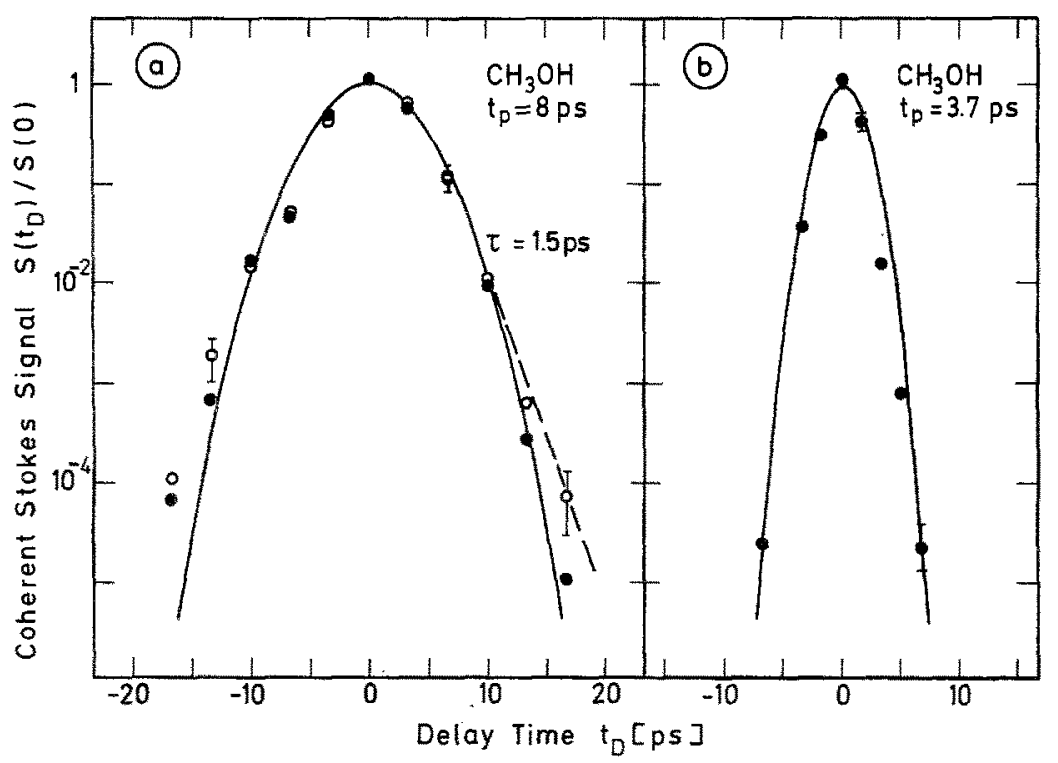

Fig, $8 a$ and $b$. Normalized coherent scattering signal as a function of the delay time in liquid $\mathrm{CH}_{3} \mathrm{OH}$. Arithmetically averaged experimental data give a larger signal in the wings (circles). The solid points are obtained from the center of the signal distribution curve. For both pulse durations, $t_{p}=8 \mathrm{ps}$ and $t_{p}=3.7 \mathrm{ps}$, the experimental points lie close to a Gaussian function (solid curves) which represents the correlation curve of the applied laser pulse first experiment we used the generator set-up of Fig. 1a with a cell length of $5 \mathrm{~cm}$. We measured the coherent probe scattering for two different pulse durations. Figure $8 \mathrm{a}$ shows data when long pulses of 8 ps were used. We obtained the open circles by averaging (arithmetic means) over approximately 30 individual measurements for each delay position. In this case, a symmetric shape of the probing signal is obtained with exponentially decaying wings (dashed line). The same result was obtained for both Raman bands of methanol. One is tempted to deduce a decay time of $1.5 \mathrm{ps}$ from the data points in the wings of the signal curve. Before commenting on this time constant, we wish to discuss the effect of the variation of the pulse durations of the exciting laser pulses [23]. We determined the probability distribution of the 30 individual measurements at each delay position. It was found that the arithmetic mean does not coincide with the center of the probability distribution but is located at higher values (for small signal values in the wings). The center values of the various distributions are plotted as solid points in Fig. 8. These points are in good agreement with a Gaussian function (solid curve) which represents the shape of the laser pulses. We conclude from this investigation that the open circles (the apparent exponential decay) result from the arithmetic averaging procedure and are of no physical significance. Working with considerable shorter laser pulses of $t_{p}=3.7 \mathrm{ps}$ we find a much shorter rise and decay of the scattered Stokes signal. The plotted points correspond to the center of the probability distribution; they fit quite well a Gaussian function. It is interesting to note that the curves of the scattered signal in Fig. $8 a$ and $b$ have the same shape as the corresponding autocorrelation functions measured with the help of a nonlinear crystal. In other words, Fig. $8 \mathrm{a}$ and $\mathrm{b}$ give information on the pulse properties but say nothing about the dephasing time of the excited vibrational mode.

Coherent probe scattering of liquid acetone gave very similar results. Again, the shape of the laser pulse was found when working with a small Stokes conversion.

A change of the signal curve in Fig. $8 \mathrm{~b}\left(t_{p} \approx 3.7 \mathrm{ps}\right)$ was observed for high Stokes conversion and longer cells; i.e., for saturation of the exciting laser pulse. In this case the signal curve becomes broader and the data points - obtained from the arithmetic mean - give exponentially decaying wings which suggest a time constant of approximately $1.5 \mathrm{ps}$. These wings disappear when the points corresponding to the center of the distribution function are plotted. The experiments demonstrate that coherent probing of inhomogeneously broadened transitions gives no information on vibrational dephasing times.

Several remarks should be made concerning earlier publications on the same subject [8-12]. According to the new observations presented here, the interpretation of certain previous data has to be revised. Since the probing experiments do not provide a selectivity for a small molecular subgroup, one must see the rapid destructive interference of a broad group of molecules. The exponential wings of the signal curve are caused by variations of the pulse durations and the averaging procedure of the data points. We note, that we are able to find similar time constants at high Stokes conversion which were reported in previous publications.

\section{Conclusions}

In this paper coherent Raman probe scattering was reconsidered and the potential of measuring phase- 
relaxation processes in the time domain of picoseconds was discussed. For single homogeneous vibrational transitions the dephasing time $T_{2}$ can be determined directly from the decay of the scattered probe signal. The spectra of the scattered probe pulse show a distinct time dependent frequency shift resulting from the nonresonant susceptibility of the medium. Of interest is the narrow probe spectrum taken during the free relaxation of the molecules. Multiple equidistant homogeneously broadened transitions give rise to a beating phenomenon resulting from the interference of the various vibrational components. The beating period gives the frequency difference between the various transitions and the signal decay provides the dephasing time $T_{2}$. The duration of the exciting and probing pulses strongly influences the shape of the signal curve. For longer pulses the beating pattern is completely obscured. Transitions with a band of continuously distributed frequencies show a rapid decay of the total material excitation due to the destructive interference of the various vibrational components. The determination of the dephasing time of a molecular subgroup does not appear to be possible with existing experimental instrumentation. Probing experiments with inhomogeneously broadened transitions are useful for the determination of the shape of the laser pulse.

\section{References}

1. A.Laubereau, W. Kaiser: Rev. Mod. Phys. 50, 607 (1978)

2. A.Laubereau, D. von der Linde, W. Kaiser: Phys. Rev. Lett. 28, 1162 (1972)

A.Laubereau, D. von der Linde, W.Kaiser: Opt. Commun. 7, 173 (1973)
3. R. R. Alfano, S.L. Shapiro: Phys. Rev. Lett. 29, 1655 (1972)

4. A.Laubereau, D. von der Linde, W.Kaiser: Phys. Rev. Lett. 27, 802 (1971)

D. von der Linde, A.Laubereau, W.Kaiser: Phys. Rev. Lett. 26, 954 (1971)

5. A.Laubereau, G.Wochner, W.Kaiser: Opt. Commun. 14, 75 (1975)

6. A.Laubereau: Chem. Phys. Lett. 27, 600 (1974)

7. S. A.Akhmanov: In Proc. International School of Physics Enrico Fermi, Course LXIV, Nonlinear Spectroscopy (North-Holland, Amsterdam 1977)

8. A.Laubereau, G.Wochner, W.Kaiser: Phys. Rev. A13, 2212 (1976)

9. A.Laubereau, G.Wochner, W.Kaiser: Chem. Phys. 28, 363 (1978)

10. C.B.Harris, H. Auweter, S. M.George: In Picosecond Phenomena $I I$, ed. by R.M.Hochstrasser, W.Kaiser, C.V.Shank, Springer Ser. Chem. Phys. 14 (Springer, Berlin, Heidelberg, New York 1980) p. 151

11. S.M.George, H.Auweter, C.B.Harris: J. Chem. Phys. 73, 5573 (1980)

12. C.B.Harris, H.Auweter, S.M.George: Phys. Rev. Lett. 44, 737 (1980)

13. S.L.McCall, E.L.Hahn: Phys. Rev. 183, 457 (1969)

14. W.Zinth, A.Laubereau, W.Kaiser: Opt. Commun. 22, 161 (1977)

15. J. A.Giordmaine, W.Kaiser: Phys. Rev. 144, 676 (1966) M.Maier, W.Kaiser, J.A.Giordmaine: Phys. Rev. 177, 580 (1969)

16. A.Penzkofer, A.Laubereau, W.Kaiser: Prog. Quantum Electron. 6, 55 (1979)

17. W.Zinth, A.Laubereau, W.Kaiser: Opt. Commun. 26, 457 (1978)

18. W.Zinth, W.Kaiser: Opt. Commun. 32, 507 (1980)

19. W.Zinth: Opt. Commun. 34, 479 (1980)

20. J.Mostowski, M.G.Raymer: Opt. Commun. 36, 237 (1981)

21. R.W.Hellwarth: Prog. Quantum Electron. 5, 1 (1977)

22. D. von der Linde, A.Laubereau: Opt. Commun. 3, 279 (1971)

23. W.Zinth: To be published

24. R.L.Carman, F.Shimizu, C.S.Wang, N.Bloembergen: Phys. Rev. A2, 60 (1970)

S.A.Akhmanov, K. N. Brabovich, A.P.Sukhorukov, A.S.Chirikin: Sov. Phys. Jetp 32, 266 (1971) 